\title{
Correction to: Opicapone: A Review in Parkinson's Disease
}

\author{
Lesley J. Scott ${ }^{1}$
}

Published online: 21 November 2017

(C) Springer International Publishing AG, part of Springer Nature 2017

Correction to: Drugs (2016) 76:1293-1300

https://doi.org/10.1007/s40265-016-0623-y

Page 1293, column 2, section 1, paragraph 1, lines 1-3: the following sentence, which previously read:

Parkinson's disease (PD) is the most common chronic, progressive, neurodegenerative disease, with a mean age of onset of 57 years $[1,2]$.

Should read:

Parkinson's disease (PD) is the second most common chronic, progressive, neurodegenerative disease, with a mean age of onset of 57 years [1,2].

Page 1294, column 1, paragraph 2, lines 1-6: the following sentence, which previously read:

Only a small fraction of an administered L-Dopa dose crosses the BBB, reflecting the extensive distribution of L-Dopa and its rapid peripheral metabolism by aromatic $L$ amino acid decarboxylase to dopamine and by catechol- $O$ methyltransferase (COMT) to $3 O$-methyldopa (3-OMD) [1-3].

Should read:

Only a small fraction of an administered L-Dopa dose crosses the $\mathrm{BBB}$, reflecting the extensive distribution of

The original article can be found online at https://doi.org/10.1007/ s40265-016-0623-y.

Lesley J. Scott

demail@springer.com

1 Springer, Private Bag 65901, Mairangi Bay, 0754 Auckland, New Zealand
L-Dopa and its rapid peripheral metabolism by aromatic $L$ amino acid decarboxylase to dopamine and by catechol- $O$ methyltransferase (COMT) to $3 O$-methyldopa (3-OMD) $[2,3]$.

Page 1296, column 1, paragraph 2, lines 1-6: the following sentence, which previously read:

Significantly $(\mathrm{p} \leq 0.009)$ more opicapone $50 \mathrm{mg} /$ day than placebo or entacapone recipients experienced improvements (i.e. minimally improved to very much improved ratings) from baseline in scores for Clinician's Global Impression of Change (PGI-C) and Patient's Global Impression of Change (PGI-C) [16].

Should read:

Significantly $(\mathrm{p} \leq 0.009)$ more opicapone $50 \mathrm{mg} /$ day than placebo or entacapone recipients experienced improvements (i.e. minimally improved to very much improved ratings) from baseline in scores for Clinician's Global Impression of Change (CGI-C) and Patient's Global Impression of Change (PGI-C) [16].

Page 1299, column 2, paragraph 1, lines 1-6: the following text, which previously read:

administered three times daily [1-3]. Entacapone is also available as a fixed-dose L-Dopa/ carbidopa/entacapone tablet [40], thereby potentially improving compliance. Tolcapone is also associated with an increased risk of hepatotoxicity (albeit very rare), which limits its use to patients who fail to respond to other COMT inhibitors [1-3]. 
Should read:

administered three times daily [3]. Entacapone is also available as a fixed-dose L-Dopa/ carbidopa/entacapone tablet [40], thereby potentially improving compliance. Tolcapone is also associated with an increased risk of hepatotoxicity (albeit very rare), which limits its use to patients who fail to respond to other COMT inhibitors [3].
Page 1299 reference list: Reference 1 , which previously read:

1. Samii A, Nutt J, Ransom RR, et al. Parkinson's disease. Lancet. 2004;2004(362):1783-93

Should read:

1. Kalia LV, Lang AE. Parkinson's disease. Lancet. 2015; (386):896-912 